\title{
BAYESIAN RECONSTRUCTION OF THE EVOLUTIONARY HISTORY AND CROSS-SPECIES TRANSITION OF VARIOLA VIRUS AND ORTHOPOXVIRUSES
}

\begin{tabular}{|r|l|}
\hline Journal: & Journal of Medical Virology \\
\hline Manuscript ID & JMV-17-6344.R1 \\
\hline Wiley - Manuscript type: & Research Article \\
\hline Complete List of Authors: & $\begin{array}{l}\text { Zehender, Gianguglielmo; University of Milan, Department of Biomedical } \\
\text { and Clinical Sciences-Infectious Diseases Section; Coordinated Research } \\
\text { Center "EpiSoMI", University of Milan } \\
\text { Lai, A.; University of Milan, Department of Biomedical and Clinical Sciences } \\
\text { 'L. Sacco' Hospital, Section of Infectious Diseases and Immunopathology } \\
\text { Veo, Carla; University of Milan, Department of Clinical Sciences 'L. Sacco' } \\
\text { Hospital, Section of Infectious Diseases and Immunopathology } \\
\text { Bergna, Annalisa; University of Milan, Department of Clinical Sciences 'L. } \\
\text { Sacco' Hospital, Section of Infectious Diseases and Immunopathology } \\
\text { Ciccozzi, Massimo; Unit of Clinical Pathology and Microbiology, University } \\
\text { Campus Bio-Medico of Rome } \\
\text { Galli, Massimo; University of Milan, Department of Clinical Sciences 'L. } \\
\text { Sacco' Hospital, Section of Infectious Diseases and Immunopathology }\end{array}$ \\
\hline \hline Keywords: & $\begin{array}{l}\text { Variola virus < Virus classification, Reservoir < Disease control, } \\
\text { Epidemiology }\end{array}$ \\
\hline & \\
\hline
\end{tabular}




\section{BAYESIAN RECONSTRUCTION OF THE EVOLUTIONARY HISTORY AND CROSS- SPECIES TRANSITION OF VARIOLA VIRUS AND ORTHOPOXVIRUSES}

Gianguglielmo Zehender ${ }^{1,2} \#$, Alessia Lai ${ }^{1}$, Carla $\mathrm{Veo}^{1}$, Annalisa Bergna ${ }^{1}$, Massimo Ciccozzi ${ }^{3}$, Massimo Galli ${ }^{1}$

${ }^{1}$ Department of Biomedical and Clinical Sciences "Luigi Sacco", University of Milan, Milan, Italy;

${ }^{2}$ Coordinated Research Center "EpiSoMI”, University of Milan, Milano, Italy;

${ }^{3}$ Unit of Clinical Pathology and Microbiology, University Campus Bio-Medico of Rome, Rome, Italy;

\# Corresponding Author:

Prof. Gianguglielmo Zehender: Department of Biomedical and Clinical Sciences "Luigi Sacco", Infectious Diseases and Immunopathology Section, University of Milan, Via G.B. Grassi 74, 20157 Milan, Italy - Phone (+39) 0250319770, Fax: (+39) 0250319768 - e-mail: gianguglielmo.zehender@unimi.it 


\section{ABSTRACT}

Variola virus (VARV), the causative agent of smallpox, is an exclusively human virus belonging to the genus Orthopoxvirus, which includes many other viral species covering a wide range of mammal hosts, such as vaccinia, cowpox, camelpox, taterapox, ectromelia and monkeypox virus.

The tempo and mode of evolution of Orthopoxviruses were reconstructed using a Bayesian phylodynamic framework by analysing 80 hemagglutinin sequences retrieved from public databases. Bayesian phylogeography was used to estimate their putative ancestral hosts. In order to estimate the substitution rate, the tree including all of the available Orthopoxviruses was calibrated using historical references dating the South American variola minor clade (alastrim) to between the XVI and XIX century.

The mean substitution rate determined by the analysis was $6.5 \times 10^{-6}$ substitutions/site/year. Based on this evolutionary estimate, the time of the most recent common ancestor of the genus Orthopoxvirus was placed at about 10,000 years before the present. Cowpox virus was the species closest to the root of the phylogenetic tree. The root of VARV circulating in the XX century was estimated to be about 700 years ago, corresponding to about $1300 \mathrm{AD}$. The divergence between West African and South American VARV went back about 500 years ago (falling approximately in the XVI century). A rodent species is the most probable ancestral host from which the ancestors of all the known Orthopoxviruses were transmitted to the other mammal host species, and each of these species represented a dead-end for each new poxvirus species, without any further interspecific spread. 


\section{INTRODUCTION}

Before it was defeated by a worldwide eradication campaign, ${ }^{1}$ smallpox was one of the most devastating infectious diseases ever confronted by humankind: it caused hundreds of millions of deaths, negatively affected the growth of populations in the Old World, led to the collapse of many civilizations in the New World after they came into contact with Europeans, and contributed to the crises of great empires. $^{2-5}$

Variola virus (VARV), the causative agent of smallpox, is a member of the Poxviridae family of large double-stranded DNA viruses whose extremely variable genomic size ranges from 130 to 375 kbp in different viral species. ${ }^{6,7}$ Of the approximately 150 genes making up the poxvirus genome, those encoding the proteins responsible for virus replication, DNA repair, RNA transcription, host range, and the structural components of the virus are mainly conserved genes located in the central region, whereas the terminal genes tend to be more variable and encode proteins involved in immune modulation, such as homologues of tumour necrosis factor or interleukin-1 beta receptors. ${ }^{8}$ Moreover, the order of the genes can be different in the genome of different poxvirus species, and the genes or entire gene families present in some viral species can lack orthologues in other members of the same genus. The first approaches to the study of poxvirus phylogeny were consequently based on the presence/absence of gene families, ${ }^{9-11}$ gene order, ${ }^{11}$ or sets of conserved genes, ${ }^{9-13}$ all of which (together with a number of phylogenetic studies) led to a similar conclusion: the speciation of Chordopoxvirinae (the subfamily of Poxviridae that includes the Orthopoxvirus genus of which VARV is a member), was the product of a process as long as the history of amniotes, and a typical example of co-evolution with their vertebrate hosts. ${ }^{14}$ However, although the majority of the species belonging to the Orthopoxvirus (OPV) genus are strictly host specific, species such as cowpox (CPXV), and monkeypox virus (MPXV) can naturally infect various mammals. ${ }^{15} \mathrm{CPXV}$ has the broadest range of hosts as it is capable of infecting humans, bovines, 
rodents and carnivorous species. The inter-specific passage of members of the OPV genus from one mammal to another has also been supported phylogenetically. ${ }^{7}$

Studies of the evolution of VARV ${ }^{7,16}$ based on the phylogenesis of conserved core genes have dated the divergence between TATV, CMLPV and VARV about 3000-4000 years ago (YA) and that the VARV strains circulating in the XX century had a common ancestor between about 500 and 1,000 YA , including two studies based on VARV sequences isolated from mummies of the XVII-XVIII centuries. $^{17,18}$ The results of these studies have left many unanswered questions. The first unambiguous descriptions of smallpox epidemics date back to at least the first century $\mathrm{AD}$ in China $^{19}$ and the second century AD in the Mediterranean area, ${ }^{2,3,20}$ and some historical records support the hypothesis that the disease first appeared between 1,500 and 1,100 BC in China and India, and during the same period in Egypt. ${ }^{1,3,21}$

It has been speculated ${ }^{22}$ that the most recent common ancestor (MRCA) of VARV, taterapox virus (TATV), and camelpox virus (CMLPV) may have been introduced into the Horn of Africa 3,5004,500 YBP by domestic Camelus dromedarius imported from the Arabian peninsula ${ }^{23,24}$ and that Gerbilliscus kempi and humans were first infected in the same area. This African origin may explain the presence of VARV in the Nile valley hypothetically causing the death of Ramses V in 1,157 BC. ${ }^{1,3}$

Various discrete trait evolution analysis models have recently been developed and used to study viral phylodynamics, which makes it possible to reconstruct the phylogenetic history, geographical origin, dispersal and migration of a virus, and has led to the discipline of phylogeography. ${ }^{25}$ One of the most interesting applications is the ancestral reconstruction of host-shifts during the evolution of a multi-host virus, which has been the subject of a number of exemplary studies. ${ }^{25,26}$

The aim of this study was to reconstruct the tempo and mode of evolution of the now extinct VARV, its relationships with other extant OPVs, the most probable time of its origin and its ancestral reservoirs using a phylogenetic/phylodynamic approach. 


\section{MATERIALS AND METHODS}

\subsection{Sequence data sets}

A total of 54 hemagglutinin (B7R) sequences of VARV, 906 nucleotides long, and sampled in known years (from 1946 to 1977) and geographical locations, were retrieved from public databases (Genbank at: http://www.ncbi.nlm.nih.gov/genbank/) and aligned with 26 different OPVs including seven camelpox (CMLPV), six monkeypox (MPXV), four cowpox (CPXV), one taterapox (TATV), one ectromelia (ECTV), and seven vaccinia viruses (VACV) including three Brazilian Cantagalo virus (CTGV), two Horsepox (HSPV), one Buffalopox (BPXV), and one Rabbitpox virus (RPXV). The sampling dates of the animal isolates ranged from 1941 (RPXV) to 2008 (one CTGV and one HSPV) .

\subsection{Phylogenetic reconstruction}

The evolutionary model that best fitted the data was selected using an information criterion implemented in JmodelTest v. 2.1.7 ${ }^{27}$ (freely available at http://darwin.uvigo.es/ software/jmodeltest.html), which selected the HKY $+\mathrm{G}$ (Hasegawa-Kishino-Yano plus Gamma) nucleotide substitution model for both data sets.

A Bayesian phylogenetic tree was constructed using the main dataset with the best-fitting substitution model chosen by JModelTest, using MrBayes. ${ }^{28}$

A Markov chain Monte Carlo (MCMC) search was made for $6 \times 10^{6}$ generations using tree sampling every 100th generation and a burn-in fraction of 50\%. Statistical support for specific clades was obtained by calculating the posterior probability of each monophyletic clade, and a posterior consensus tree was generated after a $50 \%$ burn-in.

The tree was displayed and edited using Figtree software v 1.4.1, which is freely available on the web (http://tree.bio.ed.ac.uk/software/figtree/).Maximum likelihood (ML) phylogenetic tree was inferred with PhyML program ${ }^{29}$, using the same substitution model. The statistical robustness and 
reliability of the branching order within each phylogenetic tree were confirmed with the bootstrap analysis.

The evolutionary rates were estimated using a Bayesian Markov Chain Monte Carlo (MCMC) method implemented in BEAST 1.8.0, ${ }^{30}$ under strict and relaxed (with an uncorrelated log normal rate distribution) clock conditions. As coalescent priors, parametric demographic models of population growth (constant size, exponential growth and logistic growth) and a piecewise-constant Bayesian skyline plot (BSP) ${ }^{31}$ were compared. In order to be more confident in our results, two different calibration approaches were used to estimate the substitution rates: the first was based on heterochronous sampling dates; the second was based on anthropological/historical data, in particular, the coalescence of the South American variola minor clade was independently calibrated by assuming that this strain was imported to America not before the first slave trades and not after 1,860 , when clinical alastrim was first described. To this end, a normally distributed prior with a mean value of 250 years and a standard deviation (SD) of 50 were used.

The chains were run for 100 million generations until reaching convergence, and sampled every 10,000 steps. Convergence was assessed on the basis of the effective sampling size (ESS $=>200$ ) after a 10\% burn-in using Tracer software v. 1.5 (http://tree.bio.ed.ac.uk/software/tracer/).

Uncertainty in the estimates was indicated by the $95 \%$ highest posterior density (95\% HPD) intervals, and the best fitting models were selected using a Bayes factor (BF) with marginal likelihoods implemented in BEAST. In accordance with Kass and Raftery, the strength of the evidence against $\mathrm{H} 0$ was evaluated as follows: $2 \operatorname{lnBF}<2$ no evidence; $2-6$ weak evidence; $6-10$ strong evidence, and $>10$ very strong evidence. A negative $2 \operatorname{lnBF}$ indicates evidence in favour of H0. Only values of $\geq 6$ were considered significant. A less restrictive BSP (a non-parametric piecewise-constant model) was used as the coalescent prior. The trees were summarised in a maximum clade credibility (MCC) tree (the tree with the largest product of posterior clade probabilities) after a $10 \%$ burn-in using the Tree Annotator program included in the BEAST package. The time of the most recent common ancestor (tMRCA) estimates were expressed as mean 
and 95\% HPD years before the most recent sampling dates, corresponding to 2008 for the analysed dataset.

\subsection{Discrete trait analysis: phylogeography and host shifts}

The discrete trait diffusion analysis for estimating host species transitions was made using the continuous-time Markov chain (CTMC) process over discrete sampling locations implemented in BEAST $1.80^{32}$ for discrete trait analysis. The Bayesian stochastic search variable selection (BSSVS) model was implemented in order to allow diffusion rates to be zero with a positive prior probability. Comparison of the posterior and prior probabilities that the individual rates would be zero provided a BF for testing the significance of the linkages between locations: rates with a BF of $>3$ were considered well supported and as forming the migration pathway. The chains were run for 300 million generations until reaching convergence, and sampled every 30,000 steps.

Since only a single rabbitpox virus sequence was available and the natural host of the virus is not definitely known, RPXV was excluded from the analysis.

In order to estimate the direction of the diffusion rates between hosts, an asymmetrical (nonreversible) substitution model for transition was also used. ${ }^{33}$

The final maximum clade credibility (MCC) tree was visualised using FigTree version 1.4.1. The significant migration rates were analysed and visualised using SPREAD (available at: http://www.kuleuven.be/aidslab/phylogeography/SPREAD.html) and SPREAD3 (available at: https://rega.kuleuven.be/cev/ecv/software/SpreaD3_tutorial).

The hemagglutinin sequences were assigned to groups corresponding to the known host/reservoir of the viral species. In particular, five groups were built: rodent (including TATV, CPXV, ECTV, MPXV), human (including VARV), camel (including CMLPV), bovine (including, CTGV and BPXV) and equine (HSPV).

The MacClade v 4 program (Sinauer Associates, Sunderland, MA) was used on the global dataset and the subset of VARV sequences to test viral gene inflow/outflow among different hosts using a modified version of the Slatkin and Maddison test as described below. ${ }^{34}$ The gene flow analysis was 
made by classifying the sequences into the same groups previously described (human, camel, equine, bovine and rodent). A one-character data matrix was obtained from the original data set by assigning a one-letter code to each taxon in the tree to indicate its group of origin. The putative origin of each ancestral sequence (i.e. internal node) in the tree was then inferred by finding the most parsimonious reconstruction (MPR) of the ancestral character. The final tree length (i.e. the number of observed migrations in the genealogy) was compared with the tree-length distribution of 10,000 trees, after random joining and splitting. Observed genealogies that are significantly shorter than random trees indicate the presence of subdivided populations with restricted gene flow. Specific migrations among different areas (character states) were traced using the state changes and stasis tool (MacClade software). 


\section{RESULTS}

\subsection{Tempo and mode of VARV and OPV evolution}

\subsubsection{Phylogenetic analysis}

The Bayesian (Fig. 1) and maximum likelihood trees (data not shown) of the Poxvirus dataset $(n=80)$ showed many significant clades whose high bootstrap and posterior probability values corresponded to the different viral species (VARV, VACV, CPXV, CMLPV, TATV, ECTV and MPXV). VACV and MPXV tended to segregate from the other viruses, forming a significant subclade (A) with a CPXV as the outgroup (bootstrap value=1,000; posterior probability, $p p=1$ ), whereas the other viruses grouped in a single major clade (B) with the ECTV strain as the outgroup (bootstrap value $=1,000 ; p p=1$ ). The VARV clade (bootstrap value $=1,000 ; p p=1$ ) branched into two lineages: the first, $\mathrm{P} 1$ (bootstrap value $=625 ; \mathrm{pp}=1$ ), included isolates from all over the Old World (Africa, Asia, Europe), whereas the second, P2 (bootstrap=682; pp=0.94), only included viruses that were isolated or originated in America (alastrim) and West Africa to form two highly significant subclades (bootstrap values $=683$ and $840 ; p p=0.98$ and 1 ).

Three significant subclades were identified within lineage P1: the first (bootstrap $=640 ; p p=0.98$ ) including isolates from Middle East, India and one isolate from former Yugoslavia that were obtained between 1962 and 1972 (ME/IN in Fig. 1); the second (bootstrap=660; pp=0.90) included Japanese strains isolated between 1946 and 1951, and a single Indian strain obtained in 1953 (FE/IN in Fig. 1); and the third (bootstrap=863; $p \mathrm{p}=1$ ), included all of the isolates from Bangladesh sampled in 1974, which grouped with a single Nepalese isolate sampled one year before (IN in Fig. $1)$.

\subsubsection{Estimated evolutionary rate and dated tree reconstruction}

The Bayes factor comparison of the coalescent models showed that the model best fitting the data set was a coalescent prior BSP under a strict clock (2lnBF strict $v s$ relaxed clock $=23.62$ ). The estimate of the substitution rate based on incorporating the putative calibration point in the South American variola minor MRCA provided a mean substitution rate of $6.5 \times 10^{-6}$ with a confidence 
interval of 1.2-12.6 $\times 10^{-6} \mathrm{subs} / \mathrm{site} /$ year. The calibration of the tree by heterochronous sequences gave very similar values, with a mean $7.1 \times 10^{-6}$ subs/site/year, but with broader interval $\left(95 \% \mathrm{HPD}=0.2-17 \times 10^{-6}\right)$. For this reason we adopted only the estimates obtained by historical calibration.

Table 1 summarises the main clades and the tMRCA estimates for the internal nodes with $95 \%$ HPDs obtained by historical calibration (Fig S1). The mean tree-root tMRCA was 9,507 YA (95\%HPD: 20572-2445 YA). Among the animal poxviruses, the oldest were cowpox (3,328 YA) and VACV (1,790 YA). The estimated root of the clade corresponding to VARV was 720 YA, with cumulative credibility limits of 251 and 1,470 YA. VARV subclade P1 had a tMRCA of 406 YA (95\%HPD: 849-121 YA), and P2 had a tMRCA of 508 YA (95\%HPD: 984-200 YA), thus suggesting that both subclades originated between the late XV and the early XVI century. The tMRCA of the American alastrim, used to calibrate the tree was estimated to be 277 YA (95\%HPD: 364-185 YA), corresponding to the XVIII century. The internal node connecting the long branches bringing to VARV, TATV and CMLPV, showed a $\mathrm{pp}=0.77$ and dated a mean of 4,351 YA $(95 \% \mathrm{HPD}=19238$ 1135 YA).

\subsubsection{Discrete trait analysis for host transitions}

Bayesian discrete trait analysis (Fig. 2, panel A), performed on 79 isolates with the exclusion of RPXV, showed that the most probable host at the root-tree and the backbone of the phylogenetic tree was rodent (state posterior probability, spp, 0.95). In particular, it was inferred that rodents were the ancestral hosts of clades A and B, and that of VARV ( $\mathrm{spp}=0.93$ ). All the other hosts were apparently dead-end hosts (at the external nodes of the tree).

The BSVSS analysis showed a total of 5 non-zero rates $(95 \%$ HPD=4-7), with highly supported linkages between rodent and camel $(\mathrm{BF}=12.3)$, rodent and human $(\mathrm{BF}=7.1)$, rodent and bovine $(\mathrm{BF}=3.1)$, and bovine and equine $(\mathrm{BF}=1695)$ (Fig.3). 
The Bayesian asymmetrical diffusion model showed highly supported linkages from rodent to camel $(\mathrm{BF}=16.1)$, to human $(\mathrm{BF}=11.3)$, to bovine $(\mathrm{BF}=6.5)$; and reciprocally from bovine and equine $(\mathrm{BF}=14.3)$ and vice versa $(\mathrm{BF}=3.7)$.

The migration analysis is shown Fig. 2, panel B. The null hypothesis of panmixia (i.e. no population subdivision or complete intermixing of sequences from different geographical areas) was rejected by the randomisation test $(\mathrm{P}<0.0001)$.

The gene flow analysis, which was made using a modified version of the Slatkin and Maddison test, showed significant gene flows $(50 \%$ each) from rodent to human and to camel. No significant flows between non-rodent hosts or from other hosts to rodent were found by either the maximum parsimony model. 


\section{DISCUSSION}

VARV has been extinct in nature since the end of the 1970s. The full-length genome sequences available in public databases were obtained from the few viral strains still circulating between the 1940s and late 1970s, and their scarcity greatly limits the reconstruction of VARV's evolutionary history. However, the other poxviruses still infecting different mammals (including humans) can be used to infer the evolutionary history of VARV.

Two of the main studies aimed at reconstructing the temporal scale of VARV evolution were based on phylogenetic analyses of sequences encompassing the conserved central viral genomic region. ${ }^{7,22}$ Hughes et al. postulated that the divergence between variola major and variola minor occurred 7001,000 YA, and that the common ancestor of VARV, TATV and CMLPV dated back to about 3,0004,000 YA. Babkin and Babkina, who used the unclassified Yoka poxvirus as the outgroup, obtained that the separation between TATV, VARV and CMLPV occurred about 3,500 YA and the most recent VARV strain radiated about 1,000 YA. Similar estimates (mean tMRCA of VARV clade about 1,000 YA) were obtained from a phylogenetic analysis of partially amplified B7R, A30L and E9L sequences from an ancient Siberian mummy buried in the Yakutian permafrost and dated to between the end of XVII and the beginning of the XVIII century. ${ }^{17}$ Despite the differences in the approaches used, the viral genes considered, and the sequences included in these studies, the proposed dating of VARV and estimated substitution rates (from about $2 \times 10^{-6}$ to $6 \times 10^{-6}$ sub/site/years) were substantially similar.

On the contrary a recent study based on viral sequences amplified from a Lithuanian mummy dated to middle of the XVII century ${ }^{18}$ estimated a more rapid rate of evolution of between 7.3 and $9.6 \mathrm{x}$ $10^{-6}$ nucleotide substitutions/site/year (mean: $8.5 \times 10^{-6} \mathrm{subs} / \mathrm{site} / \mathrm{year}$ ), and allowed the authors to place the origin of VARV between 1588 and 1645.

Interestingly, in both of the studies of viral sequences obtained from mummies, the position of the ancient isolates in the dated tree was always at the outgroup of the VARV clade (external to both P1 and P2 subclades). ${ }^{35}$ This could have been due to the fact that all of the smallpox isolates included 
in the analysis were sampled over a relatively narrow period of time (between the 1940s and the late 1970s) that represents only a small interval of time in the long history of smallpox virus diversification ${ }^{36}$ and immediately before its extinction. In these conditions the use of a "short-term" calibration (such as the methods based on heterochronous sequences) might overestimate the substitution rates and underestimate the tMRCAs. It has been suggested that long-term calibration using "fossil" records or archeological data is more suitable for calibrating trees with distantly correlated taxa. The inclusion in the present study of VARV and other animal OPVs extends the time-scale of the evolutionary process considered to thousands of years, thus making calibration with external data more suitable than the internal calibration approach. ${ }^{37}$

It is well known that alastrim, the less deadly American variant of VARV, was first described in the Caribbean region in the $1860 \mathrm{~s}^{1}$ and that it definitely could not have been present in the Americas before the arrival of Europeans. Accordingly, the South American MRCA was calibrated using a prior approximation of between 150 and 500 years ago, which led to an estimated mean of $6.5 \times 10^{-6}$ subs/site/year for the hemagglutinin sequences, thus making the date of the tree-root tMRCA of animal OPVs about 10,000 YA, and that of the ancestor of VARV about 1,000 YA. The use of the internal calibration based on sampling dates, gave a mean substitution rate estimate similar but with a largest interval of confidence. The time estimates described in the present study are in line with previous data, ${ }^{16}$ but do not provide any reason for the smallpox epidemics that occurred before the year 1,000 AD, strongly supported by the historical literature and archeological findings. ${ }^{38}$

It should be noticed that the incidence of smallpox decreased in Europe after vaccination was introduced, and that all of the smallpox cases seen in Europe and North America after the first World War were imported, mainly from Asia, ${ }^{38,39}$ including those involved the last two European outbreaks..$^{39,40}$ The oldest sequences available in public databases come from isolates that were obtained not before the 1940s, when the European strains of VARV were already extinct, and not representative of the autochthonous European and North-American strains. 
This supports the hypothesis that smallpox occurred in the Old World in successive waves, with the epidemics caused by distinct but related strains. Historical data testify that smallpox tended to lose its virulence in affected populations, becoming a childhood disease that reappeared cyclically and led to major outbreaks whenever the number of susceptible children reached a critical threshold in the Mediterranean area and Middle East during the Middle Ages. ${ }^{1,41}$ This was probably also the process that led to the reduced virulence of alastrim. ${ }^{1}$ On the contrary, the occurrence of major outbreaks in places where smallpox had been endemic for a long time (as evidenced by several examples in XVI and XVII centuries) suggests the possibility of the emergence of new and more virulent strains. ${ }^{1,42}$ Moreover, between the XVII and the end of the XVIII century, mortality due to smallpox significantly increased in many parts of Europe, ${ }^{1,43}$ thus suggesting that something had changed in the circulating VARV.

It therefore seems that various strains appeared and disappeared during the long evolution of human VARV. One of the aims of this study was to reconstruct the ancestral host transitions of the different OPV species on the basis of a phylogenetic diffusion model applied to a Bayesian framework. Some of the OPV species in the Old World had a number of strictly host-specific species, whereas others such as CPXV and MPXV had a broad range of hosts. The findings of phylogenetic studies suggest the existence of at least two CPXV species, both of which have the largest genomes of all OPVs (about $360 \mathrm{kbp}),{ }^{44,45}$ and CPXVs can infect humans, cows, rats, cats, dogs, and zoo animals, including elephants and large felines. ${ }^{6,46,47}$ Serological evidence and the findings of experimental studies suggest that it can be carried by wild rodent species, including bank voles (Myodes glareolus), field voles (Microtus agrestis) and striped field mice (Apodemus sylvaticus), ${ }^{48-50}$ and it has been isolated in a root vole (Microtus oeconomus) in northern Russia. ${ }^{51}$ Natural CPXV infection has recently been documented in a common vole (Microtus arvalis) trapped in Germany, ${ }^{52}$ but it has only been isolated in wild rodents in western Eurasia, a geographic range that is more restricted than of the rodent species that can be infected by $\mathrm{CPXV} .{ }^{48}$ It can therefore be presumed that the natural hosts of CPXV are wild rodents. 
Monkeypox virus (MPXV) was first isolated from captive non-human primates in Denmark, ${ }^{53}$ and subsequently from a wild squirrel (Funisciurus anerythrus) in Zaire in $1985^{54}$ and a juvenile sooty mangabey (Cercocebus atys) in Côte d'Ivoire. ${ }^{55}$ Serological evidence of MPXV infection has also been documented in various African rodents, including Cricetomys gambianus, Funisciurus spp., Heliosciurus spp., Graphiurus kelleni, Mastomys natalensis and Steatomys parvus. ${ }^{56,57}$

Two other OPVs have only been isolated in rodents: ECTV has been observed in laboratory mice (Mus spp) but almost nothing is known regarding its natural distribution, ${ }^{58,59}$ and TATV, a close relative of VARV, was isolated in naked-soled gerbil (Tatera kempi) captured in Benin in $1975 .^{60}$ Vaccinia group include viruses which were originally used for vaccine development, and do not correspond to the actually circulating Cowpox virus. In 1939 Allan Downie showed that vaccinia and cowpox viruses have different characteristics, suggesting that they were not the same virus. In particular, based on phylogenetic studies, we currently know that VACV is more closely related to horsepox than to cowpox virus. ${ }^{61}$ Horsepox has a wide spread infection among horses, causing sporadic infection in humans, and now is rare or eliminated in Europe and North America. ${ }^{62,15}$ Other Vaccinia-like viruses infecting different animals were buffalopox virus (BPXV), causing diseases in Asian buffalos and occasionally zoonotic infections in humans ${ }^{15}$ and a virus causing outbreaks among cattle and dairy workers in Brazil, called Cantagalo virus. ${ }^{63}$ Finally, a rabbitpox virus has been isolated from laboratory but not from wild rabbits, ${ }^{15}$ and it is not definitely known if rabbits are the real natural hosts of the virus. ${ }^{64}$ For this reason we excluded from our phylogeographic analysis of the host transitions the single rabbitpox virus isolate that was available on public databases at the moment when the study was performed.

All the VACV-like virus grouped in a single highly significant clade $(\mathrm{pp}=1)$ dating a mean of 1789 YA $(95 \% \mathrm{HPD}=414-3946 \mathrm{YA})$ and the ancestral host resulted more significantly a bovine (stp= 0.77), rather than an equine $(\mathrm{stp}=0.23)$. Nevertheless, an host shift to horse occurred, in our estimates, 500-600 YA, with an upper limit of about 400 YA, thus suggesting that HPXV existed at the time in which Jenner developed the vaccine. 
Assigning the analysed isolates to five groups (rodents, humans, camels, equines and bovines), rodents resulted in this work the most probable hosts in all of the deeper (ancestral) nodes of the tree, including the root. All of the other host species were at terminal nodes, which suggests that these cross-species transmissions are mainly dead ends in the evolutionary history of the virus. In our reconstruction, the analysis of the linkages between hosts by means of asymmetrical models confirmed that the rates from rodents to all of the other hosts were significant.

These results, which were also confirmed by maximum parsimony analysis, support the role of rodents as a reservoir of OPVs and the view that the other hosts in which a viral jump was successful were unable to sustain further host transition. It is possible that the profound gene rearrangement needed to adapt a new host species was a one-way process in the majority of cases, thus preventing a return to the less specialised characteristics of the strains with a broad range of hosts. CPXV has the longest genome and the largest number of genes conditioning host range: ${ }^{45}$ twenty-seven have been recognised in its genome, as against the 15 of $\mathrm{VARV}^{45}$ and the closely related CMLPV and TATV. ${ }^{15}$ It has been hypothesised that the loss of genes during evolution typical of Poxviruses is part of viral adaptation to a new host. The elimination of genes that are not essential to maintaining infection causes the emergence of new viral species that are only capable of infecting a limited number of animal hosts, and frequently only one. It has been suggested CPXV is the common ancestor of all $\mathrm{OPVs}^{7}$ and, more recently, more than $25 \%$ for VACV infection has been found in rodents captured in Brazil, ${ }^{65}$ once again suggesting the possible role of rodents as a reservoir.

The present study confirmed the previous hypothesis ${ }^{16}$ that the common ancestor of Old World OPVs was an ancestral CPXV-like virus infecting rodents which was capable of being efficiently transmitted to a wide range of susceptible hosts, including humans. The spillover event triggering the successful adaptation to humans may be dated back to between about 4,000 YA (when the common ancestor of the group VARV+TATV+CMLPV existed) and about 1,000 YA (when the radiation of the available VARV strains began in humans). The long evolutionary history of 
adaptation to new human hosts can explain both the loss of unnecessary genes and the subsequent extinction of strains with different levels of virulence. 


\section{REFERENCES}

1. Fenner F. Smallpox and its eradication. WHO. 1988.

2. Littman RJ, Littman ML. Galen and the Antonine plague. AJP. 1973;94:243-255.

3. Hopkins DR. Princes and Peasants: Smallpox in History. University of Chicago Press; 1983.

4. Fears JR. The plague under Marcus Aurelius and the decline and fall of the Roman Empire. Infect Dis Clin N Am. 2004;18:65-77.

5. Gourevitch D. The galenic plague: a breakdown of the imperial pathocoenosis. Pathocoenosis and longue duree. Hist Phil Life Sci. 2005;27:57-69.

6. Essbauer S, Pfeffer M, Meyer H. Zoonotic poxviruses. Vet Microbiol. 2010;140:229-236.

7. Hughes AL, Irausquin S, Friedman R. The evolutionary biology of poxviruses. Infect Genet Evol. 2010;10:50-59.

8. Seet BT, Johnston JB, Brunetti CR, et al. Poxviruses and immune evasion. Annu Rev Immunol. 2003;21:377-423.

9. Gubser C, Hue S, Kellam P, Smith GL. Poxvirus genomes: a phylogenetic analysis. J Gen Virol. 2004;85:105-117.

10. Hughes AL, Friedman R. Poxvirus genome evolution by gene gain and loss. Mol Phylogenet Evol. 2005;35:186-195.

11. Xing K, Deng R, Wang J, Feng J, Huang M, Wang X. Genome-based phylogeny of poxvirus. Intervirology. 2006;49:207-214.

12. McLysaght A, Baldi PF, Gaut BS. Extensive gene gain associated with adaptive evolution of poxviruses. PNAS. 2003;100:15655-15660.

13. Bratke KA, McLysaght A. Identification of multiple independent horizontal gene transfers into poxviruses using a comparative genomics approach. BMC Evol Biol. 2008;8:67.

14. Fenner F, Kerr PJ. Evolution of the poxviruses, including the coevolution of virus and host in myxomatosis. The evolutionary biology of viruses. Raven Press, New York. 1994:273-292. 
15. Haller SL, Peng C, McFadden G, Rothenburg S. Poxviruses and the evolution of host range and virulence. Infect Genet Evol. 2014;21:15-40.

16. Babkin IV, Babkina IN. The origin of the variola virus. Viruses. 2015;7:1100-1112.

17. Biagini $\mathrm{P}$, Theves $\mathrm{C}$, Balaresque $\mathrm{P}$, et al. Variola virus in a 300 -year-old Siberian mummy. $N$ Engl J Med. 2012;367:2057-2059.

18. Duggan AT, Perdomo MF, Piombino-Mascali D, et al. 17th Century Variola Virus Reveals the Recent History of Smallpox. Curr Biol. 2016;26:3407-3412.

19. Wong KC, Wang C, Wu L. History of Chinese Medicine: Being a Chronicle of Medical Happenings in China from Ancient Times to the Present Period. Tientsin Press. 1936.

20. Miller G. The Adoption of Inoculation for Smallpox in England and France. University of Pennsylvania Press. 1957.

21. Dixon CW. Smallpox. Churchill. 1962.

22. Babkin IV, Babkina IN. A retrospective study of the orthopoxvirus molecular evolution. Infect Genet Evol. 2012;12:1597-1604.

23. Bulliet RW. The Camel and the Wheel. CUP. 1975.

24. Fagan BM, Beck C. The Oxford companion to archaeology. OUP. 1996.

25. Streicker DG, Turmelle AS, Vonhof MJ, Kuzmin IV, McCracken GF, Rupprecht CE. Host phylogeny constrains cross-species emergence and establishment of rabies virus in bats. Science. 2010;329:676-679.

26. Faria NR, Suchard MA, Rambaut A, Streicker DG, Lemey P. Simultaneously reconstructing viral cross-species transmission history and identifying the underlying constraints. Philos Trans $R$ Soc Lond B Biol Sci. 2013;368:20120196.

27. Posada D. jModelTest: phylogenetic model averaging. Mol Biol Evol. 2008;25:1253-1256.

28. Huelsenbeck JP, Ronquist F. MRBAYES: Bayesian inference of phylogenetic trees. Bioinformatics. 2001;17:754-5. 
29. Guindon S, Dufayard Jf Fau - Lefort V, Lefort V Fau - Anisimova M, Anisimova M Fau Hordijk W, Hordijk W Fau - Gascuel O, Gascuel O. New algorithms and methods to estimate maximum-likelihood phylogenies: assessing the performance of PhyML 3.0. Syst Biol. 2010.59:307-21.

30. Drummond AJ, Suchard MA, Xie D, Rambaut A. Bayesian phylogenetics with BEAUti and the BEAST 1.7. Mol Biol Evol. 2012;29:1969-1973.

31. Drummond AJ, Rambaut A, Shapiro B, Pybus OG. Bayesian coalescent inference of past population dynamics from molecular sequences. Mol Biol Evol. 2005;22:1185-1192.

32. Lemey P, Rambaut A, Drummond AJ, Suchard MA. Bayesian phylogeography finds its roots. PLoS Comput Biol. 2009;5:e1000520.

33. Edwards CJ, Suchard MA, Lemey P, et al. Ancient hybridization and an Irish origin for the modern polar bear matriline. Curr Biol. 2011;21:1251-1258.

34. Slatkin M, Maddison WP. A cladistic measure of gene flow inferred from the phylogenies of alleles. Genetics. 1989;123:603-613.

35. Wertheim JO. Viral Evolution: Mummy Virus Challenges Presumed History of Smallpox. Curr Biol. 2017;27:R119-R120.

36. Theves C, Biagini P, Crubézy E. The rediscovery of smallpox. Clin Microbiol Infect. 2014;20:210-218.

37. Ho SY, Saarma U, Barnett R, Haile J, Shapiro B. The effect of inappropriate calibration: three case studies in molecular ecology. PLoS One. 2008;3:e1615.

38. Geddes AM. The history of smallpox. Clin Dermatol. 2006;24:152-157.

39. Wehrle PF, Posch J, Richter KH, Henderson DA. An airborne outbreak of smallpox in a German hospital and its significance with respect to other recent outbreaks in Europe. Bull World Health Organ. 1970;43:669-679. 
40. Ristanovic E, Gligic A, Atanasievska S, Protic-Djokic V, Jovanovic D, Radunovic M. Smallpox as an actual biothreat: lessons learned from its outbreak in ex-Yugoslavia in 1972. Ann Ist Super Sanita. 2016;52:587-597.

41. Greenhill WA. A Treatise on the small-pox and measles. Sydenham Society.1848.

42. Corrêa G, de Lima Felner RJ. Lendas Da India. Academia Real Das Sciencias. 1864.

43. Guy WA. Two Hundred and Fifty Years of Small Pox in London. JSTOR. 1882;45:399-443.

44. Carroll DS, Emerson GL, Li Y, et al. Chasing Jenner's vaccine: revisiting cowpox virus classification. PLoS One. 2011;6:e23086.

45. Bratke KA, McLysaght A, Rothenburg S. A survey of host range genes in poxvirus genomes. Infect Genet Evol. 2013;14:406-425.

46. Bennett M, Gaskell CJ, Baxbyt D, Gaskell RM, Kelly DF, Naidoot J. Feline cowpox virus infection. JSAP. 1990;31:167-173.

47. Baxby D, Bennett M, Getty B. Human cowpox 1969-93: a review based on 54 cases. Brit J Dermatol. 1994;131:598-607.

48. Chantrey J, Meyer H, Baxby D, et al. Cowpox: reservoir hosts and geographic range. Epidemiol Infect. 1999;122:455-460.

49. Hazel SM, Bennett M, Chantrey J, et al. A longitudinal study of an endemic disease in its wildlife reservoir: cowpox and wild rodents. Epidemiol Infect. 2000;124:551-562.

50. Kinnunen PM, Henttonen H, Hoffmann B, et al. Orthopox virus infections in Eurasian wild rodents. Vector Borne Zoonotic Dis. 2011;11:1133-1140.

51. L'Vov S D, Gromashevskii VL, Marennikova SS, Bogoiavlenskii GV, Bailuk FN. [Isolation of poxvirus (Poxviridae, Orthopoxvirus, the cowpox complex) from the root vole Microtus (M.) oeconomus Pal. 1776 in the forest tundra of the Kola Peninsula]. Vopr Virusol. 1988;33:92-94.

52. Hoffmann D, Franke A, Jenckel M, et al. Out of the Reservoir: Phenotypic and Genotypic Characterization of a Novel Cowpox Virus Isolated from a Common Vole. J Virol. 2015;89:1095910969. 
53. Magnus Pv, Andersen EK, Petersen KB, Birch-Andersen A. A POX-LIKE DISEASE IN CYNOMOLGUS MONKEYS. APMIS. 1959;46:156-176.

54. Khodakevich L, Jezek Z, Kinzanzka K. Isolation of monkeypox virus from wild squirrel infected in nature. Lancet. 1986;1:98-99.

55. Radonić A, Metzger S, Dabrowski PW, et al. Fatal Monkeypox in Wild-Living Sooty Mangabey, Côte d'Ivoire, 2012. Emerg Infect Dis. 2014;20:1009-1011.

56. Reynolds MG, Carroll DS, Olson VA, et al. A silent enzootic of an orthopoxvirus in Ghana, West Africa: evidence for multi-species involvement in the absence of widespread human disease. Am J Trop M Hyg. 2010;82:746-754.

57. Orba Y, Sasaki M, Yamaguchi $\mathrm{H}$, et al. Orthopoxvirus infection among wildlife in Zambia. $J$ Gen Virol. 2015;96:390-394.

58. Marchal J. Infectious ectromelia. A hitherto undescribed virus disease of mice. J Pathol Bacteriol. 1930;33:713-728.

59. Osterhaus AD, Teppema JS, Wirahadiredja RM, van Steenis G. Mousepox in the Netherlands. Lab Anim Sci. 1981;31:704-706.

60. Lourie B, Nakano JH, Kemp GE, Setzer HW. Isolation of poxvirus from an African Rodent. J Infect Dis. 1975;132:677-681.

61. Qin L, Favis N, Famulski J, Evans DH. Evolution of and evolutionary relationships between extant vaccinia virus strains. J Virol. 2015;89:1809-1824.

62. Damaso CR. Revisiting Jenner's mysteries, the role of the Beaugency lymph in the evolutionary path of ancient smallpox vaccines. Lancet Infect Dis. 2017.

63. Damaso CR, Esposito JJ, Condit RC, Moussatche N. An emergent poxvirus from humans and cattle in Rio de Janeiro State: Cantagalo virus may derive from Brazilian smallpox vaccine. Virology. 2000;277:439-449.

64. Chapman JL, Nichols DK, Martinez MJ, Raymond JW. Animal models of orthopoxvirus infection. Vet Pathol. 2010;47:852-870. 
65. Miranda JB, Borges IA, Campos SPS, et al. Serologic and Molecular Evidence of Vaccinia Virus Circulation among Small Mammals from Different Biomes, Brazil. Emerg Infect Dis. 2017;23:931-938. 


\section{FIGURE LEGENDS}

Fig. 1. Bayesian tree of 80 Orthopoxvirus genomes reconstructed using Mr Bayes. The asterisks on the branches represent bootstrap support (see Materials and Methods for details). The main significant clades/subclades are highlighted. The bar at the bottom indicates the nucleotide substitutions per site. The main clades (A and B) and the VARV lineages (P1 and P2) are highlighted. Vaccinia, VACV including Cantagalo virus (CTGV), Horsepox (HSPV), Buffalopox (BPXV), and Rabbitpox virus (RPXV); monkeypox virus, MPXV; cowpox virus, CPXV; Ectromelia virus, ECTV; camelpox virus, CMLPV; taterapox virus, TATV; variola virus, VARV (EU=Europe, AF=Africa, IN=India, WA=West Africa, SA=South America, EO=Far East, $\mathrm{MO}=$ Middle East $)$.

Fig. 2. Panel A shows the Bayesian phylogeographic tree of the hosts/reservoirs of the viral species. The branches are coloured on the basis of the most probable host of the descendent nodes (camel; human $=\mathrm{HU}$; bovine $=$ Bov; equine $=\mathrm{Equ}$; rodent $=\mathrm{Rod})$. The numbers on the internal nodes indicate significant state posterior probabilities $(\mathrm{spp}>0.8)$. Panel B shows the phylogeographical map. The bubblegrams indicate the frequency of the gene flows (migrations) to/from different hosts, with the area of each circle being proportional to the percentage of observed migrations in the ML genealogy. The migrations were inferred using a modified version of the Slatkin and Maddison algorithm.

Fig. 3. Significant non-zero migration rates of VARV hosts/reservoirs (camel; human=HU; bovine $=$ Bov; equine $=$ Equ; rodent $=$ Rod) supported by a $\mathrm{BF}$ of $>3$ as indicated by colour lines. The migrations were calculated using SPREAD3. The direction of migrations calculated using bayesian asymmetrical diffusion model were indicated by arrowheads. 


\section{SUPPLEMENTARY MATERIAL}

Fig. S1. The maximum clade credibility (MCC) tree of the Orthopoxviruses (vaccinia, VACV; monkeypox virus, MPXV; cowpox virus, CPXV; Ectromelia virus, ECTV; camelpox virus, CMLPV; taterapox virus, TATV; variola virus, VARV, EU=Europe, AF=Africa, IN=India, $\mathrm{WA}=$ West Africa, $\mathrm{SA}=$ South America, $\mathrm{EO}=$ Far East, $\mathrm{MO}=$ Middle East). The numbers on the internal nodes indicate significant posterior probabilities ( $\mathrm{pp}>0.7$ ), and the scale at the bottom of the tree represents calendar years. Calibration point is indicated. 
Table1. tMRCA estimates and 95\% HPDs of the main clades observed in the dated tree.

\begin{tabular}{|c|c|c|c|c|c|c|c|c|c|}
\hline \multirow[b]{2}{*}{ Clade } & \multirow[b]{2}{*}{ Host } & \multirow[b]{2}{*}{ bootstrap } & \multirow[b]{2}{*}{ pp } & \multicolumn{3}{|c|}{ Calibrated } & \multicolumn{3}{|c|}{ Year } \\
\hline & & & & tMRCA & 95\%HPDL & 95\%HPDH & mean & 95\%HPDL & 95\%HPDH \\
\hline Root & & & & 9507 & 2445 & 20572 & -7499 & -437 & -18564 \\
\hline VACV & Different & 97.8 & 0.85 & 1790 & 414 & 3947 & 218 & 1594 & -1939 \\
\hline MPXV & Rodent & 100 & 1 & 556 & 79 & 1361 & 1452 & 1929 & 647 \\
\hline CPXV & Rodent & 5.5 & 1 & 3328 & 780 & 7386 & -1320 & 1228 & -5378 \\
\hline CMLPV & Camel & 100 & 1 & 3328 & 248 & 2891 & -1320 & 1760 & -883 \\
\hline VARV & Human & 100 & 1 & 720 & 251 & 1470 & 1288 & 1757 & 538 \\
\hline P1 & & 62.5 & 1 & 406 & 121 & 849 & 1602 & 1887 & 1159 \\
\hline P2 & & 68.2 & 0.93 & 508 & 200 & 984 & 1500 & 1808 & 1024 \\
\hline P2-WAF & & 84 & 1 & 198 & 54 & 419 & 1810 & 1954 & 1589 \\
\hline P2-SA & & 68.3 & 1 & 277 & 185 & 364 & 1731 & 1823 & 1644 \\
\hline P1-ME/IN & Human & 64.0 & 0.98 & 129 & 52 & 268 & 1879 & 1956 & 1740 \\
\hline P1-FE/IN & Human & 66 & 0.99 & 103 & 62 & 183 & 1905 & 1946 & 1825 \\
\hline P1-IN & Human & 86.3 & 1 & 85 & 38 & 172 & 1923 & 1970 & 1836 \\
\hline
\end{tabular}

tMRCA, time of the most recent common ancestor; 95\% HPDL, highest posterior density low interval; $95 \%$ HPDH, highest posterior density high interval; VACV, Vaccinia,; MPXV, Monkeypox virus; CPXV, Cowpox virus; CMLPV, Camelpox virus; VARV, Variola virus; WAF, West Africa; SA, South America; ME, Middle East; FE; Far East; IN, India. 
Fig. 1. Bayesian tree of 80 Orthopoxvirus genomes reconstructed using Mr Bayes. The asterisks on the branches represent bootstrap support (see Materials and Methods for details). The main significant clades/subclades are highlighted. The bar at the bottom indicates the nucleotide substitutions per site. The main clades (A and B) and the VARV lineages (P1 and P2) are highlighted. Vaccinia, VACV including Cantagalo virus (CTGV), Horsepox (HSPV), Buffalopox (BPXV), and Rabbitpox virus (RPXV); monkeypox virus, MPXV; cowpox virus, CPXV; Ectromelia virus, ECTV; camelpox virus, CMLPV; taterapox virus, TATV; variola virus, $\operatorname{VARV}(E U=$ Europe, $A F=A f r i c a, I N=I n d i a, W A=$ West Africa, $S A=S o u t h$ America, $E O=F a r$ East, $\mathrm{MO}=$ Middle East).

\section{$113 \times 92 \mathrm{~mm}(300 \times 300 \mathrm{DPI})$}


A

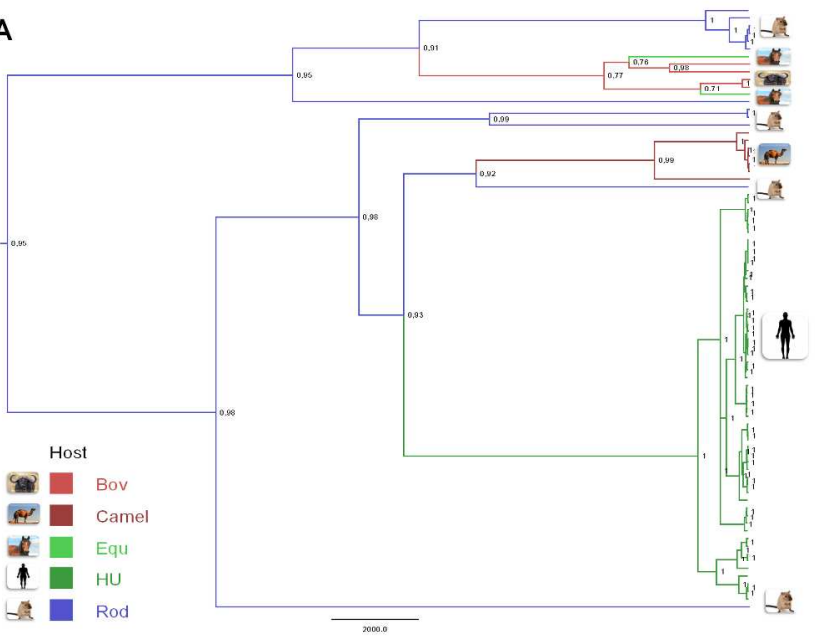

B

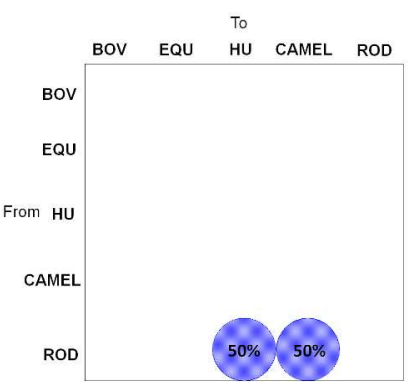

Fig. 2. Panel A shows the Bayesian phylogeographic tree of the hosts/reservoirs of the viral species. The branches are coloured on the basis of the most probable host of the descendent nodes (camel; human=HU; bovine=Bov; equine $=E q u$; rodent $=$ Rod). The numbers on the internal nodes indicate significant state posterior probabilities (spp >0.8). Panel B shows the phylogeographical map. The bubblegrams indicate the frequency of the gene flows (migrations) to/from different hosts, with the area of each circle being proportional to the percentage of observed migrations in the ML genealogy. The migrations were inferred using a modified version of the Slatkin and Maddison algorithm.

$256 \times 138 \mathrm{~mm}(300 \times 300 \mathrm{DPI})$ 


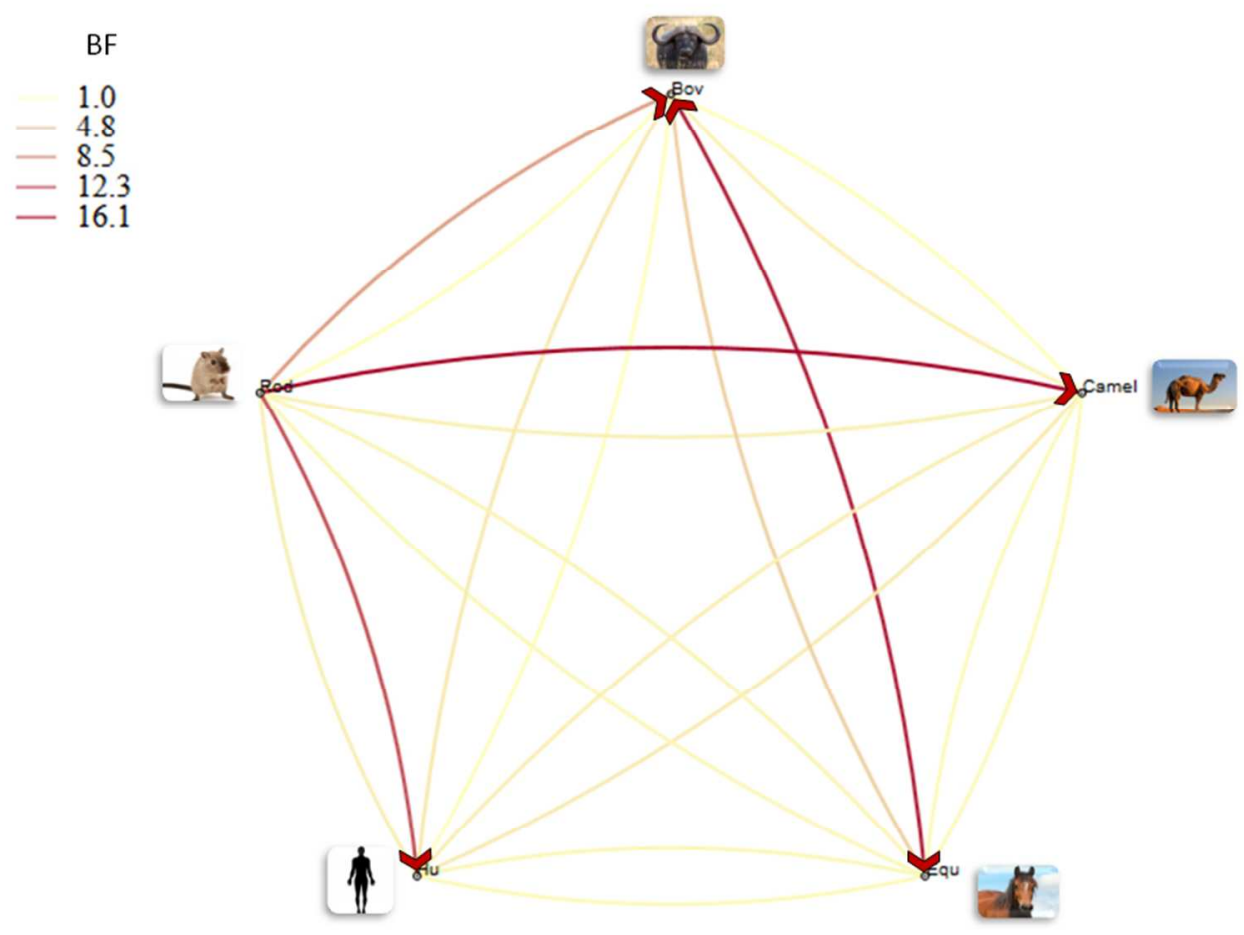

Fig. 3. Significant non-zero migration rates of VARV hosts/reservoirs (camel; human=HU; bovine=Bov; equine $=E q u$; rodent $=$ Rod) supported by a BF of $>3$ as indicated by colour lines. The migrations were calculated using SPREAD3. The direction of migrations calculated using bayesian asymmetrical diffusion model were indicated by arrowheads.

$81 \times 60 \mathrm{~mm}(300 \times 300$ DPI $)$ 